\title{
Capacity gaps in health facilities for case management of intestinal schistosomiasis and soil-transmitted helminthiasis in Burundi
}

Paul Bizimana ${ }^{1,2,3,6^{*}}$ D, Katja Polman ${ }^{4}$, Jean-Pierre Van Geertruyden ${ }^{1}$, Frédéric Nsabiyumva ${ }^{5}$, Céline Ngenzebuhoro ${ }^{6}$, Elvis Muhimpundu ${ }^{7}$ and Giuseppina Ortu ${ }^{8}$

\begin{abstract}
Background: Schistosomiasis and soil-transmitted helminthiasis (STH) are endemic diseases in Burundi. STH control is integrated into health facilities (HF) across the country, but schistosomiasis control is not. The present study aimed to assess the capacity of HF for integrating intestinal schistosomiasis case management into their routine activities. In addition, the current capacity for HF-based STH case management was evaluated.
\end{abstract}

Methods: A random cluster survey was carried out in July 2014, in 65 HF located in Schistosoma mansoni and STH endemic areas. Data were collected by semi-quantitative questionnaires. Staff with different functions at the HF were interviewed (managers, care providers, heads of laboratory and pharmacy and data clerks). Data pertaining to knowledge of intestinal schistosomiasis and STH symptoms, human and material resources and availability and costs of diagnostic tests and treatment were collected.

Findings: Less than half of the 65 care providers mentioned one or more major symptoms of intestinal schistosomiasis (abdominal pain 43.1\%, bloody diarrhoea 13.9\% and bloody stool 7.7\%). Few staff members (15.7\%) received higher education, and less than $10 \%$ were trained in-job on intestinal schistosomiasis case management. Clinical guidelines and laboratory protocols for intestinal schistosomiasis diagnosis and treatment were available in one third of the HF. Diagnosis was performed by direct smear only. Praziquantel was not available in any of the HF. The results for STH were similar, except that major symptoms were more known and cited (abdominal pain 69.2\% and diarrhoea $60 \%$ ). Clinical guidelines were available in $61.5 \%$ of HF, and albendazole or mebendazole was available in all HF.

Conclusions: The current capacity of HF for intestinal schistosomiasis and STH detection and management is inadequate. Treatment was not available for schistosomiasis. These issues need to be addressed to create an enabling environment for successful integration of intestinal schistosomiasis and STH case management into HF routine activities in Burundi for better control of these diseases.

Keywords: Capacity gap, Health facility, Case management, Intestinal schistosomiasis, Soil-transmitted helminthiasis, Burundi

\footnotetext{
* Correspondence: pbizimana2010@gmail.com; bizap2006@yahoo.fr

${ }^{1}$ Global Health Institute, Department of Epidemiology and Social Medicine,

Faculty of Medicine and Health Sciences, University of Antwerp, Gouverneur

Kinsbergencentrum, Doornstraat 331, Wilrijk, 2610 Antwerp, Belgium

${ }^{2}$ Département des Sciences de la Santé Publique, Direction de la Formation,

Institut National de Santé Publique, B.P, 6807 Bujumbura, Burundi

Full list of author information is available at the end of the article
}

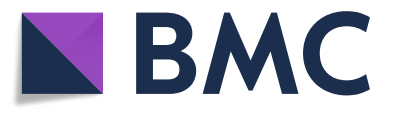

(C) The Author(s). 2018 Open Access This article is distributed under the terms of the Creative Commons Attribution 4.0 International License (http://creativecommons.org/licenses/by/4.0/), which permits unrestricted use, distribution, and

reproduction in any medium, provided you give appropriate credit to the original author(s) and the source, provide a link to the Creative Commons license, and indicate if changes were made. The Creative Commons Public Domain Dedication waiver (http://creativecommons.org/publicdomain/zero/1.0/) applies to the data made available in this article, unless otherwise stated. 


\section{Multilingual abstracts}

Please see Additional file 1 for translation of the abstract into the six official working languages of the United Nations.

\section{Background}

Among the neglected tropical diseases (NTD), schistosomiasis and soil-transmitted helminthiasis (STH) are the most common parasitic infections. Both have major implications for health and socioeconomic aspects and are important public health problems in Burundi [1].

From 1950 to the 1960 s, population movement from the highlands to the Rusizi plain, deterioration of water and sanitation infrastructures and migration of refugees from neighbouring countries contributed to a serious increase of the disease burden due to Schistosoma mansoni [2-6]. A high prevalence of STH (Ascaris lumbircoides, Trichuris trichuria, and hookworm) in Usumbura in 1935 [7] and in the Central Highlands (around Kitega) in 1936 [8] had already been reported.

In Burundi, STH case management has been integrated into health facilities (HF) across the country for many decades. This is not the case for schistosomiasis, for which any form of control only began in the 1970s. In 1973, a schistosomiasis treatment programme for schools was implemented in the capital city of Bujumbura, and ambulant treatment for positive cases was initiated at the health centres (HC). In both cases, direct smear was used for diagnosis and niridazole for treatment $[6,9]$. This resulted in a decrease of the school prevalence of $S$. mansoni infection from $16 \%$ in 1974 to $10 \%$ in 1982 [9]. With the availability of new tools for diagnosis (Kato-Katz test) and treatment (praziquantel [PZQ]) [10] of intestinal schistosomiasis, a new national control programme was launched in 1982 for intestinal schistosomiasis and STH control using Kato-Katz as the diagnostic tool and PZQ and mebendazole (MBZ) for intestinal schistosomiasis and STH treatments, respectively [9]. Ten years later, in the primary schools of Bujumbura, the prevalence had decreased from 49.5 to $29.4 \%$ for STH and from 23.3 to $6.4 \%$ for S. mansoni infection [9].

In 1989, S. mansoni control was integrated into the primary health care (PHC) services of all provinces of Burundi where the disease was endemic (Bujumbura Mairie, Bujumbura Rural, Bubanza, Cibitoke, Bururi, Makamba and Kirundo) [11] (Fig. 1). However, in 1993, the civil war began. Throughout the country, the resulting situation of instability affected all factors of life, including the health sector, and control activities declined considerably [11]. In 2005, S. mansoni and STH were again widespread, with prevalence in some provinces reaching 61 and $60 \%$, respectively [12].

In 2007, a Neglected Tropical Disease Control Programme was implemented in Burundi. The main objectives were to define populations at risk for each NTD and to develop and implement a drug treatment strategy targeting at-risk populations. A nationwide school-based survey in 20 schools confirmed that intestinal schistosomiasis and STH were still endemic in the country, with 24 communes at high risk of these diseases [13]. No urogenital schistosomiasis was found, confirming previous reports [3, 4]. During the same year, a programme of mass drug administration (MDA) with PZQ and albendazole (ALB)/MBZ was initiated. Target groups were school-age children for PZQ and children between 1 and 14 years of age and pregnant women for ALB/MBZ [13]. From 2007 to 2011, the treatment coverage in school-age children at the national level increased from $87.9 \%$ in 2008 to $95.9 \%$ in 2011 [13].

Data from a countrywide reassessment of S. mansoni infection in Burundi published in 2017 showed a significant decrease in the prevalence from $12.7 \%$ in 2007 to $2.2 \%$ in 2014 [14] in communes targeted by MDA [14]. For STH, a significant decrease was also registered from 2007 to 2014 [15]. The pooled STH prevalence was $32 \%$ in 2007 and $18 \%$ in 2014 [15].

In Burundi, STH control is integrated into HF across the country, but schistosomiasis control is not, with only $\mathrm{HC}$ involved and only during MDA campaigns. Vertical programmes are difficult to maintain over long periods, especially when they are dependent on external funding $[16,17]$. For sustainability reasons, the World Health Organization (WHO) 1993 recommendation on integrating schistosomiasis control into PHC services [18] was reconsidered by others [19] in 2010.

We investigated the capacity of HF to integrate intestinal schistosomiasis case management into their routine activities. In addition, the capacity for current HF-based STH case management was evaluated. To this end, the current knowledge of health staff on the symptoms and the available options for diagnosis and treatment of intestinal schistosomiasis and STH were assessed in endemic areas in Burundi at HF of different levels.

\section{Material and methods}

Burundi: Health system and general socioeconomic context

In Burundi, the health system consists of three levels. The central level consists of the directorate-general, departmental directorates, vertical programmes and national and specialized hospitals. The intermediate level is composed of 17 sanitary provinces (SP) corresponding to the administrative provinces. At the peripheral level, there are 45 sanitary districts (SD) with $753 \mathrm{HC}$ and 63 hospitals. Each SD includes a district hospital (DH) and many $\mathrm{HC}$. The SD covers an average of two or three communes. A commune is the most decentralized and operational unit 


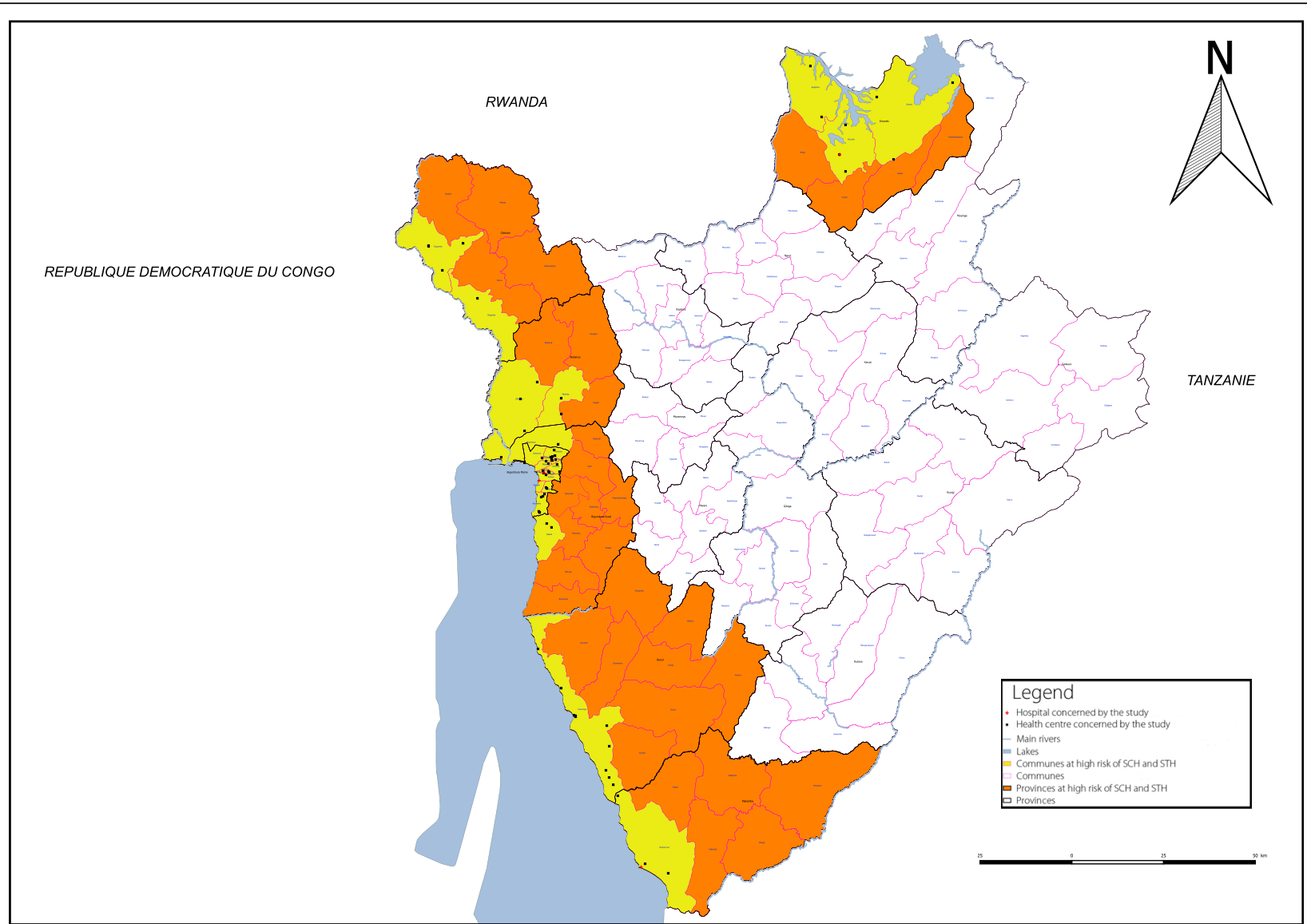

Fig. 1 Burundi map indicating the location of the 65 health facilities included in the study, Burundi 2014. SCH: Schistosomiasis; STH: Soil-transmitted helminthiasis.

of Burundi's administrative system. Burundi had $129 \mathrm{com}$ munes in 2014, at the time of the survey. The size of a commune was $216 \mathrm{~km}^{2}$ (average), with an average population of 71978 inhabitants per commune. The HC is the first patient contact point in the health system and is generally managed by a nurse. The heads of the SP, SD and $\mathrm{DH}$ are medical doctors.

Most of the Burundian population live in precarious socioeconomic conditions, with 8 out of 10 living below the poverty level (less than 1 USD/day) [20]. The per capita daily income is 0.64 USD and 0.41 USD in urban and rural areas, respectively. The gross domestic product (GDP) fell dramatically from 286 USD/capita in 1993 to 176 USD/ capita in 2011 [21]. Eighty percent of the population live less than $5 \mathrm{~km}$ from $\mathrm{HC}$; however, 17\% of patients do not have access to care, and $81.5 \%$ of patients are obliged to borrow money or sell assets to cope with health/medical expenses [20]. Furthermore, only 55\% of households have access to clean water in rural areas and $85 \%$ in urban areas [20], while only $12.6 \%$ use latrines or pit latrines [20]. Sixty-one percent of the $\mathrm{HC}$ and $27 \%$ of the primary schools have tap water. Ninety percent of the population attend primary school, but only 17 and $24 \%$ of girls and boys, respectively, complete the first level of secondary school, and only 9 and $17 \%$ complete the second level [21].

\section{Study area}

This study was conducted in 24 communes endemic for intestinal schistosomiasis and STH in Burundi. These communes are located in the following seven provinces: Bujumbura Mairie, Bujumbura Rural, Bubanza, Bururi, Cibitoke, Kirundo and Makamba. These provinces are located in the west, south and north of Burundi. All are close to lakes and rivers separating Burundi from the Democratic Republic of Congo in the west (Lake Tanganyika and the Rusizi River), Tanzania in the south and east (Maragarazi River) and Rwanda in the north (Lake Cohoha and Lake Rweru) (Fig. 1).

\section{Study design}

This study was designed to assess the knowledge of health staff on the symptoms and the available options for diagnosis and treatment of intestinal schistosomiasis 
and $\mathrm{STH}$ in endemic areas of Burundi. While the whole country is endemic for STH infections, only 24 communes are considered to be at risk of intestinal schistosomiasis infection (per 2007 mapping) [13]. Based on these data, 65 HF were randomly selected from a list of $220 \mathrm{HF}$ located in those 24 communes endemic for intestinal schistosomiasis and STH. Selection was performed via proportional allocation to strata (communes), ensuring that at least one HF in each commune was randomly selected.

\section{Questionnaires and data collection}

In each of the $65 \mathrm{HF}$, five staff were interviewed: the person in charge of the HF (manager), the person in charge of consultations and referral for patients (care provider), the person in charge of the laboratory (head of laboratory), the person in charge of the pharmacy (head of pharmacy), and the person in charge of case reporting (data clerk). The interviewers were medically trained people. A pre-survey was carried out in six HC (not included in the final randomization of the main survey) in the 13 communes of Bujumbura Mairie to test the questionnaires in terms of formulation and understanding of the questions.

The survey was conducted in July 2014, from the 5th to 12th and was organized into 3 axes and nine teams with, respectively, three supervisors and nine team leaders, supervised by one coordinator. The questions posed to the HF staff focused on (i) HF type (public, confessional and private) and level (national hospital, regional hospital, $\mathrm{DH}$ and $\mathrm{HC}$ ) in the Burundian health pyramid; (ii) knowledge and education/training of staff (degree/diploma and position held, in-job training on intestinal schistosomiasis and STH and knowledge of symptoms related to intestinal schistosomiasis and STH); (iii) diagnosis (use and availability of clinical guidelines, laboratory protocols and diagnostic tests for intestinal schistosomiasis and STH); (iv) treatment (availability, purchasing and supply process and the list of essential medicines); (v) costs (consultation fees and prices for diagnosis and treatment); and (vi) case reporting activities (monitoring of patients on treatment and reporting of diagnosis and treatment to the National Health Information System [NHIS]).

\section{Definition of some degrees/diplomas used in the questionnaires in the Burundian context}

$\mathrm{A} 0=$ Secondary school +4 years of higher education (higher education is defined as the superior level that follows secondary school; secondary school = high education); $\mathrm{A} 1=$ Secondary school +3 years of higher education; $\mathrm{A} 2=$ Primary school +8 years of secondary school; A3 = Primary school +6 years of secondary school.

\section{Statistical analysis}

Databases were created in Excel 2013 (Microsoft, Redmond, United States of America) and exported to Stata version 12 (StataCorp. LP, College Station, United States of America) for statistical analysis. Data are shown in tables and graphs with calculated frequencies (with 95\% confidence intervals for proportions in Fig. 2) and mean \pm standard deviation (or median and interquartile range (IQR) when the distribution was abnormal) for qualitative and quantitative variables, respectively. Chi square and Fisher's exact tests were used to analyse the association of the knowledge of care providers on different symptoms of schistosomiasis and STH with their level of education, using an $\alpha$ risk error of $5 \%(P<0.05)$.

\section{Results}

\section{General characteristics of HF}

Among the $65 \mathrm{HF}$ selected, 61 were $\mathrm{HC}(94 \%)$ and four were hospitals. Among the $61 \mathrm{HC}, 22$ (33.9\%) were public, nine (13.9\%) confessional and $30(46.2 \%)$ private. Among the four hospitals, two (3\%) were public district hospitals, one was a public National Hospital (1.5\%) and one was private (1.5\%).

\section{Human resources \\ Education and job type of staff}

Table 1 gives an overview of the education and job type of the HF staff. Managers (43.1\%) and care providers (46.2\%) were mainly A2 nurses, while heads of pharmacy (36.9\%) were mainly A3 nurses. For data clerks, the proportions of A2 and A3 nurses were equal (38.5\%). Heads of laboratory were mainly A2 laboratory technicians (52.3\%).

Across all levels, the number of staff with a higher education was very low. Only $15.7 \%$ had a higher education (A1, A0, pharmacist and medical doctor), while $76.6 \%$ had secondary education in health sciences, mainly A2 and A3 nurses.

\section{Training on intestinal schistosomiasis and STH}

Only 8.1 and $9.2 \%$ of the interviewees received training on intestinal schistosomiasis and STH, respectively. Less than $50 \%$ of trained staff received the training material to be used for reference in the field- 38.1 and $45.8 \%$ for intestinal schistosomiasis and $\mathrm{STH}$, respectively. The duration of the training ranged from one to seven days, except for the laboratory technicians, who were trained for up to 21 days. In more than $98 \%$ of cases, both STH and schistosomiasis training was given simultaneously.

\section{Knowledge of symptoms of intestinal schistosomiasis and STH by care providers}

Figure 2 shows all reported symptoms for intestinal schistosomiasis and STH. The knowledge level of care 
Table 1 Education and job type of interviewees in 65 of 220 health facilities in Burundi in 2014

\begin{tabular}{|c|c|c|c|c|c|}
\hline Education and job type & $\begin{array}{l}\text { Manager of HF } \\
\text { (Percentage) }\end{array}$ & $\begin{array}{l}\text { Care Provider } \\
\text { (Percentage) }\end{array}$ & $\begin{array}{l}\text { Head of laboratory } \\
\text { (Percentage) }\end{array}$ & $\begin{array}{l}\text { Head of pharmacy } \\
\text { (Percentage) }\end{array}$ & $\begin{array}{l}\text { Data clerks } \\
\text { (Percentage) }\end{array}$ \\
\hline MD Specialist & $2(3.0 \%)$ & $0(0.0 \%)$ & $0(0.0 \%)$ & $0(0.0 \%)$ & $0(0.0 \%)$ \\
\hline MD General Practitioner & $5(7.7 \%)$ & $9(13.8 \%)$ & $0(0.0 \%)$ & $0(0.0 \%)$ & $2(3.1 \%)$ \\
\hline Pharmacist & $1(1.5 \%)$ & $0(0.0 \%)$ & $0(0.0 \%)$ & $1(1.5 \%)$ & $0(0.0 \%)$ \\
\hline Nurse AO & $2(3.1 \%)$ & $1(1.5 \%)$ & $1(1.5 \%)$ & $0(0.0 \%)$ & $2(3.1 \%)$ \\
\hline Laboratory Technician A0 & $0(0.0 \%)$ & $0(0.0 \%)$ & $5(7.7 \%)$ & $0(0.0 \%)$ & $0(0.0 \%)$ \\
\hline Midwife A0 & $0(0.0 \%)$ & $1(1.5 \%)$ & $0(0.0 \%)$ & $0(0.0 \%)$ & $1(1.5 \%)$ \\
\hline Nurse A1 & $6(9.2 \%)$ & $4(6.1 \%)$ & $0(0.0 \%)$ & $2(3.1 \%)$ & $7(10.8)$ \\
\hline Laboratory Technician A1 & $0(0.0 \%)$ & $0(0.0 \%)$ & $1(1.5 \%)$ & $0(0.0 \%)$ & $0(0.0 \%)$ \\
\hline Nurse A2 & $28(43.1 \%)$ & $30(46.2 \%)$ & $9(13.8 \%)$ & $21(32.3 \%)$ & 25 (38.5\%) \\
\hline Laboratory Technician A2 & $0(0.0 \%)$ & $0(0.0 \%)$ & $34(52.3 \%)$ & $2(3.1 \%)$ & $1(1.5 \%)$ \\
\hline Nurse A3 & $21(32.3 \%)$ & 19 (29.2\%) & $5(7.7 \%)$ & $24(36.9 \%)$ & 25 (38.5\%) \\
\hline Laboratory Technician A3 & $0(0.0 \%)$ & $0(0.0 \%)$ & $5(7.7 \%)$ & $0(0.0 \%)$ & $0(0.0 \%)$ \\
\hline Other & $0(0.0 \%)$ & $1(1.5 \%)$ & $7(10.8 \%)$ & $15(23.1 \%)$ & $2(3.1 \%)$ \\
\hline Total & 65 & 65 & 65 & 65 & 65 \\
\hline
\end{tabular}

HF Health facilities, MD Medical doctor; $A 0=$ Secondary school +4 years of higher education (higher education is defined as the superior level that follows secondary school; secondary school = high education); $\mathrm{A} 1=$ Secondary school +3 years of higher education; $\mathrm{A} 2=$ Primary school +8 years of secondary school; $\mathrm{A} 3=$ Primary school +6 years of secondary school

providers for intestinal schistosomiasis was very low. Bloody diarrhoea and bloody stools were mentioned as the main symptoms of intestinal schistosomiasis, at 13.9 and $7.7 \%$, respectively. Abdominal pain for intestinal schistosomiasis was mentioned by $43.1 \%$ of the care



providers as another important symptom. Knowledge of diarrhoea as a main symptom of intestinal schistosomiasis was related to the level of education and was significantly less among A3 diploma holders (10.5\%) compared to superior diploma holders $(50 \%)$ (Fisher's exact test $=0.004$ ).

For STH, the knowledge of symptoms was better than that for intestinal schistosomiasis. Abdominal pain was cited by $69.2 \%$ and diarrhoea by $60 \%$ of the care providers.

\section{Material resources}

Clinical guidelines and laboratory protocols

The results of our study showed limited availability of material resources for clinical and laboratory diagnosis: 40 $(61.5 \%)$ and $22(33.8 \%)$ of the 65 HF had clinical guidelines for STH and intestinal schistosomiasis, respectively. Of these HF, only eight (36.4\%) had general guidelines, and only two $(9.1 \%)$ had a diagnostic algorithm to manage STH and intestinal schistosomiasis. Laboratory protocols on intestinal schistosomiasis and STH diagnosis were only available in $19(29.2 \%)$ and 24 (36.9\%) of the HF, respectively. Among these HF, only 21 (87.5\%) and 4 (22.2\%) had written SOPs (standard operating procedures) on the direct smear test and the Kato-Katz test, respectively.

\section{Laboratory tests used for the diagnosis of intestinal schistosomiasis and STH}

In all $65 \mathrm{HF}$, only a direct smear test was available for laboratory diagnosis of intestinal schistosomiasis and STH, which was applied in $58(90.6 \%)$ and 64 (98.5\%) of the HF, respectively. The lack of some accessories was cited as one of the reasons of this situation. 


\section{Drug supply and availability of treatment}

Among the heads of pharmacy, 36 (55.4\%) reported receiving the drugs bought by HF in time, and 34 (52.3\%) receiving them in the right quantity. Only 45 (69.2\%) of the HF were allowed by the Ministry of Public Health to supply drugs of any kind. While ALB/MBZ and PZQ are on the list of essential drugs [22], this was recognized by the pharmacy staff only for ALB/MBZ, not for PZQ. In contrast to ALB/MBZ, which was present in every HF, PZQ was not available at any level. Thirty (46.2\%) of the pharmacy staff reported that PZQ should be available in the $\mathrm{HF}$, and $14(46.7 \%)$ reported having the financial means to procure it. All 65 (100\%) heads of pharmacy recognized that drugs used during the mass treatment campaigns (which are done twice and once a year for $\mathrm{STH}$ and schistosomiasis, respectively) are given by donors (Schistosomiasis Control Initiative [SCI] ranks first for drugs used for mass treatment campaigns, whether for STH or schistosomiasis).

\section{Financial accessibility to consultation, laboratory diagnosis and treatment}

Table 2 gives details on the median costs with the IQR. Consultation and diagnosis (direct smear) costs were 2 times more expensive in private $\mathrm{HC}$ than in public and confessional $\mathrm{HC}$ and 5.8 times more expensive in private hospitals than in public and confessional hospitals. The treatment for STH was 2.4 times more expensive in private $\mathrm{HC}$ than in public and confessional HC. During the MDA campaigns, medication for STH and schistosomiasis was donated. In that case, the treatment was provided free of charge in public and confessional
HC. For hospitals, the treatment was 1.3 times more expensive in private hospitals than in public and confessional hospitals.

\section{Case reporting activities}

According to the data clerks, the reporting of cases to the NHIS was functional for 63 (96.9\%) of the HF visited, both for intestinal schistosomiasis and STH. An identical paper template was used in all HC and transmitted monthly to the NHIS. The hospitals also used reporting forms, but STH was not specified. Reports were transmitted to NHIS only by the $\mathrm{DH}$, not by the national and private hospitals.

Follow-up with the patients under STH treatment was reported to be done in 49 (75.4\%) of the $65 \mathrm{HF}$. However, the follow-up visits were actually registered in only 23 of these $(46.9 \%)$. For schistosomiasis, there was no treatment available and, thus, no follow-up.

\section{Discussion}

We aimed to assess the capacity of HF to integrate intestinal schistosomiasis case management into routine activities. We also evaluated the capacity for current STH case management, which has been integrated into the HF for many decades. We found the following: (i) Few staff members had received higher education, and even less had received training on intestinal schistosomiasis case management. The level of knowledge of the main symptoms of intestinal schistosomiasis was low and was related to the level of education. (ii) Clinical guidelines and laboratory protocols were available in only one third of the HF. (iii) In HF with laboratory facilities, only direct smear was used to diagnose intestinal schistosomiasis. (iv) PZQ

Table 2 Median costs ${ }^{a}$ of schistosomiasis and soil-transmitted helminthiasis health care in 65 of 220 health facilities, Burundi 2014

\begin{tabular}{|c|c|c|c|c|}
\hline & $\begin{array}{l}\text { Public and confessional hospital } \\
\text { (IQR) }\end{array}$ & $\begin{array}{l}\text { Private hospital } \\
\text { (IQR) }\end{array}$ & $\begin{array}{l}\text { Public and confessional HC } \\
\text { (IQR) }\end{array}$ & $\begin{array}{l}\text { Private } \mathrm{HC} \\
(\mathrm{IQR})\end{array}$ \\
\hline \multicolumn{5}{|l|}{ People $\geq 5$ years of age } \\
\hline Consultation ticket $\geq 5$ years & $0.32(0.32-1.03)$ & 6.47 & $0.06(0.03-0.19)$ & $0.32(0.06-0.32)$ \\
\hline Laboratory test for the diagnosis (direct smear) & 1.13(0.65-1.13) & 1.94 & $0.26(0.19-0.32)$ & $0.32(0.26-0.32)$ \\
\hline Praziquantel tablets & - & - & - & - \\
\hline Albendazole tablets & $0.08(0.05-0.26)$ & 0.10 & $0.08(0.03-0.19)$ & $0.19(0.19-0.32)$ \\
\hline \multicolumn{5}{|l|}{ Pregnant women and children $<5$ years of age ${ }^{b}$} \\
\hline Consultation ticket & 0.00 & 6.47 & 0.00 & $0.32(0.06-0.32)$ \\
\hline Laboratory test for the diagnosis (direct smear) & 0.00 & 1.94 & 0.00 & $0.32(0.26-0.32)$ \\
\hline Praziquantel tablets & - & - & - & - \\
\hline Albendazole tablets & 0.00 & 0.10 & 0.00 & $0.19(0.19-0.32)$ \\
\hline Total costs for schistosomiasis & 1.45 & 8.41 & 0.32 & 0.64 \\
\hline Total costs for STH & 1.53 & 8.51 & 0.40 & 0.83 \\
\hline
\end{tabular}

IQR Interquartile range, $H C$ Health centre, STH Soil-transmitted helminthiasis, - Not applicable

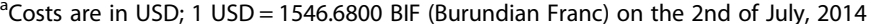

${ }^{\mathrm{b}}$ Pregnant women and children less than 5 years of age do not pay for health care in public and confessional $\mathrm{HF}$ 
was not available in any of the HF. (v) Consultation and diagnosis (based on direct smear) were relatively expensive, considering the socioeconomic conditions of Burundians. The results for STH were similar, except that (i) the level of knowledge of main symptoms was considerably higher, (ii) clinical guidelines were available in a higher proportion of the HF (61.5\%), and (iii) ALB/MBZ was available in all $\mathrm{HF}$.

The number of HF staff with higher education was very low in our study. This problem is not specific to Burundi. It is a reality in low-income countries [23] that only few HF are managed by medical doctors. For example, in Kenya [24], clinical officers in DH were reported to perform medical tasks that are typically assigned to medical doctors, including medical surgery.

Even though in-job training cannot replace prior education [25], it can bring added value in strengthening the capacity of HF for the prevention and control of infectious diseases [19, 26-32]. In Mali [33] and Senegal [34], training on schistosomiasis case management was provided as part of an intervention project on curative health care, and knowledge of health care providers about the symptoms of $S$. mansoni infections was high (69-94\%) after the training. This is not the case in Burundi, where the percentage of staff that received specific training on intestinal schistosomiasis diagnosis and treatment was very low.

Previous studies have shown that inadequate supply [35] and expired medicines and other materials [30,35], plus shortages of essential drugs, are access barriers to health services, especially in rural areas [30]. The drug supply problems identified in this study have been observed in other countries as well, such as Ethiopia [25], Uganda and Tanzania [29]. Studies in Ghana [36] and Senegal [34] reported PZQ to be out of stock in 22.5 and $25 \%$ of $\mathrm{HF}$, respectively, obliging patients to be referred to other HF or to seek treatment elsewhere. In Burundi, PZQ was unavailable at any HF, causing a major constraint for integration of schistosomiasis case management into HF [37]. Similarly, the lack of adequate diagnostic tests has also been observed in other countries [33,36] and can seriously hamper intestinal schistosomiasis and STH detection and management in HF. The WHO recommends using Kato-Katz testing for the diagnosis of intestinal schistosomiasis [18] and STH, which is more sensitive than direct smear and still relatively cheap [18]. Recent research has reported the circulating cathodic antigen test in urine as a more sensitive test $[14,38,39]$ for intestinal schistosomiasis, in addition to being easy to use. However, it is more expensive than the Kato-Katz test [40].

In Burundi, the average per capita daily income is below the poverty level (1USD): 0.64 USD per day and 0.41 USD per day in urban and rural areas, respectively [21]. Our results show that the costs of consultation and diagnosis for intestinal schistosomiasis/STH range from 0.32 to 8.41 USD, depending on the HF type and level. Hence, many Burundians cannot access care without sacrificing other needs. At present, only pregnant women and children under five years of age do not pay for health care in public or confessional HF. To remove the financial barrier to health care, this should be extended to other vulnerable groups as well.

\section{Conclusions}

This study highlighted the lack of capacity in Burundian HF for integrating intestinal schistosomiasis case management into their routine activities. For STH, the capacity for HF-based case management was better, but it still needs improvement. Especially at the $\mathrm{HC}$ level, which is the first point of contact with the community, there is an urgent need for the government to address these capacity gaps in order to provide adequate diagnosis and treatment for intestinal schistosomiasis and STH for everybody at all times. At present, treatment of intestinal schistosomiasis is limited to MDA for school-age children [13], and PZQ is not available outside of the yearly campaigns or for other target groups, jeopardizing accessibility and equity of services [19]. In addition, diagnosis in HF of both intestinal schistosomiasis and STH is currently based on direct smears in the context of poor knowledge of symptoms among care providers and, thus, is sub-optimal. As a consequence, many cases are not diagnosed and/or not treated, causing the spread of these infections and potential severe complications in the long term. HF capacity strengthening for case management is an essential, albeit not the only, requirement for sustainable control of intestinal schistosomiasis and STH in Burundi and elsewhere.

\section{Additional file}

Additional file 1: Multilingual abstracts in the six official working languages of the United Nations. (PDF 648 kb)

\section{Abbreviations \\ ALB: Albendazole; BIF: Burundian Franc; DH: District Hospital; GDP: Gross Domestic Product; HC: Health Centre; HF: Health Facility; IQR: Interquartile Range; MBZ: Mebendazole; MD: Medical Doctor; MDA: Mass Drug Administration; NHIS: National Health Information System; NTD: Neglected tropical diseases; P: P-value; PHC: Primary Health Care; PZQ: Praziquantel; SCH: Schistosomiasis; SCl: Schistosomiasis Control Initiative; SD: Sanitary District; SOP: Standard Operating Procedure; SP: Sanitary Province; USD: United States dollar; WHO: World Health Organization}

\section{Acknowledgements}

The authors would like to thank the interviewers, team leaders and supervisors who participated in the data collection. The authors are also very thankful to the different people at different positions in the health centres and hospitals visited who agreed to answer the questions of this study. The authors appreciate the technical support of the technician who drew the map of Burundi. The authors appreciated also the Director of the Schistosomiasis Control Initiative, Prof Alan Fenwick, for supporting this project 


\section{Funding}

The Schistosomiasis Control Initiative (SCI)/Imperial College and VLIR-UOS funded this study.

\section{Availability of data and materials}

The datasets generated and analysed during the current study are available from the corresponding author on reasonable request.

\section{Authors' contributions}

PB was involved in the acquisition, analysis and interpretation of data for the study and wrote the paper. GO made substantial contributions to the conception of the study, was involved in the acquisition, analysis, and interpretation of data, critically revised the manuscript and approved the final version to be published. KP made substantial contributions to the conception of the study, analysed and interpreted data and revised the manuscript critically. JPVG was involved in the conception of the study, analysis and interpretation of data and revision of the manuscript. FN, EM and CN were involved in the acquisition and analysis of data and approved the final version to be published. All authors read and approved the final manuscript.

\section{Ethics approval and consent to participate}

The research protocol was reviewed and approved by the National Ethics Committee of Burundi in September 2013. Authorisation from the Ministry of Public Health for the study was provided in May 2014.

\section{Competing interests}

The authors declare that they have no competing interests.

\section{Author details}

${ }^{1}$ Global Health Institute, Department of Epidemiology and Social Medicine, Faculty of Medicine and Health Sciences, University of Antwerp, Gouverneur Kinsbergencentrum, Doornstraat 331, Wilrijk, 2610 Antwerp, Belgium. 2Département des Sciences de la Santé Publique, Direction de la Formation, Institut National de Santé Publique, B.P, 6807 Bujumbura, Burundi. ${ }^{3}$ Département de Médecine Communautaire, Faculté de Médecine de Bujumbura, Université du Burundi, Bujumbura, Burundi. ${ }^{4}$ Medical Helminthology Unit, Department of Biomedical Sciences, Institute of Tropical Medicine, Antwerp, Belgium. ${ }^{5}$ Département de Médecine Interne, Faculté de Médecine de Bujumbura, Université du Burundi, Bujumbura, Burundi. ${ }^{6}$ Département des Sciences de la Santé Publique, Institut Universitaire des Sciences de la Santé et de Développement Communautaire, Bujumbura, Burundi. ${ }^{7}$ Programme National Intégré de Lutte contre les Maladies Tropicales Négligées et la Cécité, Département des programmes de santé, Ministère de la Santé Publique et de la Lutte contre le Sida, Bujumbura, Burundi. ${ }^{8}$ Department of Infectious Diseases and Epidemiology, Schistosomiasis Control Initiative, Imperial College, London, UK.

\section{Received: 13 December 2017 Accepted: 31 May 2018}

\section{Published online: 04 July 2018}

\section{References}

1. Tchuente LA, N'Goran EK. Schistosomiasis and soil-transmitted helminthiasis control in Cameroon and cote d'Ivoire: implementing control on a limited budget. Parasitology. 2009;136(13):1739-45.

2. Gryseels B. Intestinal schistosomiasis in the Ruzizi Valley (Burundi): preliminary studies. Ann Soc Belg Med Trop. 1984;64(3):249-66. (in French)

3. Gryseels B. Distribution of Biomphalaria and the transmission of Schistosoma in the Ruzizi Valley, Burundi: preliminary study. Ann Soc Belg Med Trop. 1985;65(1):49-58. (in French)

4. Gryseels B, Nkulikyinka L, Kabahizi E, Maregeya E. A new focus of Schistosoma mansoni in the highlands of Burundi. Ann Soc Belg Med Trop. 1987 Sep;67(3):247-57.

5. Gryseels B, Nkulikyinka L. The distribution of Schistosoma mansoni in the Rusizi plain (Burundi). Ann Trop Med Parasitol. 1988;82(6):581-90.

6. Gryseels B. The epidemiology of schistosomiasis in Burundi and its consequences for control. Trans R Soc Trop Med Hyg. 1991;85(5):626-33.

7. F.de Bève. Bilhariziose en Ruanda-Urundi et spécialement à Usumbura. Annale de la Société Belge de Médecine Tropicale. 1935;XV:3-18.

8. Neujan G. Le parasitisme intestinal chez les indigènes des Hauts Plateaux(environs de Kitega). Annale de la Société Belge de Médecine Tropicale. 1937;XVII:343-9.
9. Engels D, Ndoricimpa J, Nahimana S, Gryseels B. Control of Schistosoma mansoni and intestinal helminths: 8-year follow-up of an urban school programme in Bujumbura, Burundi. Acta Trop. 1994;58(2):127-40.

10. WHO. The control of schistosomiasis. World Health Organ Tech Rep Ser. 1985;728:1-113.

11. Engels D, Sindayigaya B, Gryseels B. Sustainability of schistosomiasis case detection based on primary health care. Trans R Soc Trop Med Hyg. 1995; 89(6):599.

12. Baza D. Rapport d'enquête sur la situation épidémiologique de la bilharziose dans les écoles primaires des provinces sanitaires endémiques du Burundi.Programme de Lutte contre les Maladies Transmissibles et Carentielles, Ministère de la Santé Publique, Burundi, 44p. 2005.

13. Ndayishimiye O, Ortu G, Soares Magalhaes RJ, Clements A, Willems J, Whitton J, et al. Control of neglected tropical diseases in Burundi: partnerships, achievements, challenges, and lessons learned after four years of programme implementation. PLoS Negl Trop Dis. 2014;8(5):e2684.

14. Ortu G, Ndayishimiye O, Clements M, Kayugi D, Campbell CH Jr, Lamine MS, et al. Countrywide reassessment of Schistosoma mansoni infection in Burundi using a urine-circulating cathodic antigen rapid test: informing the National Control Program. Am J Trop Med Hyg. 2017;96(3):664-73.

15. Ortu G, Assoum M, Wittmann U, Knowles S, Clements M, Ndayishimiye $\mathrm{O}$, et al. The impact of an 8-year mass drug administration programme on prevalence, intensity and co-infections of soil-transmitted helminthiases in Burundi. Parasit Vectors. 2016;9(1):513.

16. Mills A. Mass campaigns versus general health services: what have we learnt in 40 years about vertical versus horizontal approaches? Bull World Health Organ. 2005;83(4):315-6.

17. Murray CJ, Frenk J. A framework for assessing the performance of health systems. Bull World Health Organ. 2000;78(6):717-31.

18. WHO. The control of schistosomiasis. World Health Organ Tech Rep Ser. 1993;830:1-86.

19. Gyapong JO, Gyapong M, Yellu N, Anakwah K, Amofah G, Bockarie M, et al. Integration of control of neglected tropical diseases into health-care systems: challenges and opportunities. Lancet. 2010;375(9709):160-5.

20. Observatoire National des Ressources Humaines en Santé du Burundi. Profil des ressources humaines en santé du Burundi. Ministère de la Santé Pubmique et de la Lutte contre le Sida, Edition 2011. Burundi, Bujumbura 2012.

21. Banque Africaine de Développement-Fonds Africain de Développement. Burundi:Document de stratégie développement pays 2012-2016. Departement Régional East-A, Octobre 2011.

22. Ministère de la Santé Publique et de la Luttre contre le Sida. Ordonnance ministérielle $\mathrm{N}^{\circ} 630 / . . / . . / 2012$ portant révision de la liste nationale des médicaments essentiels du Burundi du 2 octobre 2012. Burundi, Bujumbura, octobre, 2012

23. Mendis S, Al BI, Dissanayake L, Varghese C, Fadhil I, Marhe E, et al. Gaps in capacity in primary care in low-resource settings for implementation of essential noncommunicable disease interventions. Int J Hypertens. 2012; 2012:584041.

24. Peter Gray I, Carter JY. An evaluation of clinical services laboratory in subsaharan Africa.Ex africa semper aliquid Novi? Clin Chim Acta. 1997;267:103-28.

25. Abreha T, Alemayehu B, Tadesse Y, Gebresillassie S, Tadesse A, Demeke L, et al. Malaria diagnostic capacity in health facilities in Ethiopia. Malar J. 2014;13:292.

26. Celletti F, Wright A, Palen J, Frehywot S, Markus A, Greenberg A, et al. Can the deployment of community health workers for the delivery of HIV services represent an effective and sustainable response to health workforce shortages? Results of a multicountry study. AIDS. 2010;24(Suppl 1):S45-57.

27. Katabarwa MN, Habomugisha P, Richards FO Jr, Hopkins D. Communitydirected interventions strategy enhances efficient and effective integration of health care delivery and development activities in rural disadvantaged communities of Uganda. Tropical Med Int Health. 2005;10(4):312-21.

28. Lewin S, Lavis JN, Oxman AD, Bastias G, Chopra M, Ciapponi A, et al. Supporting the delivery of cost-effective interventions in primary healthcare systems in low-income and middle-income countries: an overview of systematic reviews. Lancet. 2008:372(9642):928-39.

29. Lewin SA, Dick J, Pond P, Zwarenstein M, Aja G, Van WB, et al. Lay health workers in primary and community health care. Cochrane Database Syst Rev. 2005;1 CD004015

30. Manafa O, McAuliffe E, Maseko F, Bowie C, MacLachlan M, Normand C. Retention of health workers in Malawi: perspectives of health workers and district management. Hum Resour Health. 2009;7:65. 
31. Mendis S, Johnston SC, Fan W, Oladapo O, Cameron A, Faramawi MF. Cardiovascular risk management and its impact on hypertension control in primary care in low-resource settings: a cluster-randomized trial. Bull World Health Organ. 2010;88(6):412-9.

32. Ndyomugyenyi R, Kabatereine N. Integrated community-directed treatment for the control of onchocerciasis, schistosomiasis and intestinal helminths infections in Uganda: advantages and disadvantages. Tropical Med Int Health. 2003:8(11):997-1004.

33. Landoure A, van der Werf MJ, Traore M, de Vlas SJ. Evaluation of case management in the integrated schistosomiasis-control programme in Mali. Ann Trop Med Parasitol. 2003:97(7):723-36

34. van der Werf MJ, Mbaye A, Sow S, Gryseels B, de Vlas SJ. Evaluation of staff performance and material resources for integrated schistosomiasis control in northern Senegal. Tropical Med Int Health. 2002;7(1):70-9.

35. Makaula P. Bloch P, Banda HT, Mbera GB, Mangani C, de SA, et al. Primary health care in rural Malawi - a qualitative assessment exploring the relevance of the community-directed interventions approach. BMC Health Serv Res. 2012;12:328.

36. van der Werf MJ, Bosompem KM, de Vlas SJ. Schistosomiasis control in Ghana: case management and means for diagnosis and treatment within the health system. Trans R Soc Trop Med Hyg. 2003;97(2):146-52.

37. Hotez PJ, Engels D, Fenwick A, Savioli L. Africa is desperate for praziquantel. Lancet. 2010;376(9740):496-8.

38. Colley DG, Binder S, Campbell C, King CH, Tchuem Tchuente LA, N'Goran EK, et al. A five-country evaluation of a point-of-care circulating cathodic antigen urine assay for the prevalence of Schistosoma mansoni. Am J Trop Med Hyg. 2013;88(3):426-32.

39. Lamberton PH, Kabatereine NB, Oguttu DW, Fenwick A, Webster JP. Sensitivity and specificity of multiple Kato-Katz thick smears and a circulating cathodic antigen test for Schistosoma mansoni diagnosis pre- and post-repeatedpraziquantel treatment. PLoS Negl Trop Dis. 2014;8(9):e3139.

40. Worrell CM, Bartoces M, Karanja DM, Ochola EA, Matete DO, Mwinzi PN, et al. Cost analysis of tests for the detection of Schistosoma mansoni infection in children in western Kenya. Am J Trop Med Hyg. 2015;92(6):1233-9.

\section{Ready to submit your research? Choose BMC and benefit from}

- fast, convenient online submission

- thorough peer review by experienced researchers in your field

- rapid publication on acceptance

- support for research data, including large and complex data types

- gold Open Access which fosters wider collaboration and increased citations - maximum visibility for your research: over $100 \mathrm{M}$ website views per year

At BMC, research is always in progress.

Learn more biomedcentral.com/submissions 